RECyT

Año 22 / No 33 / 2020 / 38-46

\title{
Simulación del proceso de producción del cumeno mediante el simulador ChemCAD ${ }^{\circledR}$
}

\section{Simulation of the cumene production process by using ChemCAD ${ }^{\circledR}$ simulator}

\author{
Amaury Pérez Sánchez ${ }^{1}$ * , Elizabeth Ranero González ${ }^{1}$ Eddy. J. Pérez Sánchez² ${ }^{2}$ Norlem Liaño Abascal ${ }^{1}$ \\ 1- Facultad de Ciencias Aplicadas, Universidad de Camagüey "Ignacio Agramonte Loynaz". Carretera Circunvalación \\ Norte, Km. 51⁄2, e/ Camino Viejo de Nuevitas y Ave. Ignacio Agramonte, Camagüey, Cuba. CP 74650. \\ 2- Dirección Comercial, Empresa Servicios Automotores S.A., Calle C, e/ Abraham Delgado y Marcial Gómez, Ciego de Ávila, Cuba. \\ *E-mail: amauryps@nauta.cu \\ Recibidio el 10 de agosto de 2019, Aprobado el 6 de noviembre de 2019.
}

\section{Resumen}

\begin{abstract}
En el presente trabajo se realizó la simulación del proceso de producción del cumeno a partir de la alquilación del benceno con propileno mediante el simulador ChemCAD ${ }^{\circledR}$ versión 5.2.0. Se obtuvieron las curvas de calor de cada uno de los intercambiadores de calor de tubo y coraza utilizados, mientras que se calculó la composición y caudal de cada una de las corrientes involucradas en el proceso. También se efectuó un estudio de sensibilidad con el fin de conocer la influencia de la presión de operación del tanque flash sobre la cantidad de cumeno a obtener por la corriente del fondo de este equipo. Se obtienen $13403,54 \mathrm{~kg} / \mathrm{h}$ de cumeno por el tope de la segunda columna de destilación (columna de cumeno), con una pureza del $98,6 \%$, siendo el benceno $(1,2 \%$ la principal impureza encontrada en esta corriente. El tanque flash deberá operar a una presión de 7 atm para obtener el mayor rendimiento de cumeno en este equipo.
\end{abstract}

Palabras clave: Análisis de sensibilidad; ChemCAD ${ }^{\circledR}$; Cumeno; Rendimiento; Simulación..

\section{Abstract}

In the present work, the simulation of the cumene production process was carried out from the benzene alkylation with propylene by using the ChemCAD ${ }^{\circledR}$ simulator version 5.2.0. Heat curves of each shell and tube heat exchanger were obtained. At the same time, the composition and mass flowrate of each stream involved in the process were calculated. A sensitivity study was also carried out in order to know the influence of the flash vessel operating pressure on the amount of cumene to be obtained by the stream at the bottom of this equipment. $13403.54 \mathrm{~kg} / \mathrm{h}$ of cumene are obtained at the top stream of the second distillation column (cumene column), with a purity of $98.6 \%$, being benzene (1.2\%) the main impurity found in this stream. The flash vessel must operate at a pressure of $7 \mathrm{~atm}$ to obtain the best yield of cumene in this equipment.

Keywords: Sensitivity analysis; ChemCAD ${ }^{\circledR}$; Cumene; Yield; Simulation.

\section{Introducción}

El cumeno es un compuesto químico importante que se utiliza fundamentalmente como intermediario en la fabricación de fenol, acetona y $\alpha$-metilestireno [1]. En la actualidad el cumeno es producido mediante la alquilación del benceno con propileno utilizando un catalizador ácido como el cloruro de aluminio, trifluoruro de boro, ácido fosfórico, fluoruro de hidrógeno o zeolitas [2]. También se ha reportado la utilización de un catalizador de $\mathrm{Ni} / \gamma-\mathrm{Al}_{2} \mathrm{O}_{3}$ en un reactor de lecho fijo [3] y el empleo de bentonita con pilares de aluminio intercambiada con cationes $\mathrm{K}^{+}, \mathrm{La}^{3+}$ y $\mathrm{Al}^{3+}$ [4]. El cumeno se puede obtener además a partir de la hidroalquilación del benceno con acetona utilizando catalizadores bifuncionales conteniendo un sistema binario de óxido de cobre-cromito de cobre como el componente hidrogenado, y zeolita BEA, MOR, FAU o MFI como el componente de alquilación [5], así como también a partir de la lignina en un proceso de tres etapas en el cual se incluye la pirolisis catalítica de la lignina para obtener una mezcla de compuestos aromáticos, seguido de la desalquilación de estos compuestos aromáticos para obtener una mezcla de compuestos aromáticos ricos en benceno, y la posterior transformación de estos últimos compuestos en cumeno a través de reacciones en fase líquida utilizando catalizadores líquidos iónicos [6].

El proceso de producción del cumeno a través de la alquilación del benceno con propileno ha sido estudiado 
por varios autores. En este sentido, Luyben [7] llevó a cabo un diseño económicamente óptimo de una planta de producción de cumeno considerando costos capitales, costos de la energía y costos de las materias primas, para desarrollar una estructura de control a escala de planta industrial capaz de manejar, de forma efectiva, posibles alteraciones en la tasa de producción. En otro estudio, Norouzi y col. [8] demostraron la validez de la aplicación del Método de Superficie de Respuesta para efectuar la optimización de las condiciones operacionales del proceso de producción del cumeno a escala industrial, asi como también efectuaron la introducción de un procedimiento por pasos para encontrar las mejores condiciones de operación con mayor atracción desde el punto de vista económico. También Gera y col. [9] aplicaron el procedimiento de diseño de control a escala de planta planteado por Skogestad [10] al proceso de producción del cumeno, utilizando un análisis en estado estacionario "fluctuante" para seleccionar las variables controladas primarias "auto-optimizadas" las cuales, al mantenerse constantes, conducen a pérdidas económicas aceptables sin necesidad de re-optimizar el proceso cuando ocurran alteraciones. Por último, Mahapatra [2] simuló el proceso de producción del cumeno mediante el simulador Aspen Plus ${ }^{\circledR}$, obteniendo los resultados de balance de masa y energía de cada una de las corriente que intervienen en el proceso.

La simulación de procesos asistida por computadora se ha convertido en una herramienta estándar para planificar, optimizar, diseñar y evaluar procesos químicos. Involucra usualmente la preparación de un diagrama de flujo de proceso, la solución de los balances de masa y energía basados en la estimación de parámetros suministrados por el usuario, la estimación del costo total y el análisis de sensibilidad ante cambios en las suposiciones iniciales realizadas. La simulación de procesos proporciona un enlace entre el descubrimiento de un compuesto químico novedoso y las etapas iniciales del desarrollo de procesos, guiando esfuerzos para optimizar el proceso de fabricación de forma expedita. Resulta particularmente efectivo cuando se emplea en las etapas tempranas del desarrollo del proceso [11].

Los simuladores de procesos ofrecen la oportunidad de reducir el tiempo requerido para el desarrollo del sistema o proceso bajo estudio. Permiten comparar alternativas de proceso sobre una base consistente para que puedan sintetizarse y analizarse un gran número de ideas de forma interactiva en un corto período de tiempo [12]. Entre los simuladores más utilizados en la actualidad se encuentra el ChemCAD ${ }^{\circledR}$, el cual ha sido empleado para simular diferentes procesos productivos, entre los cuales se encuentran la fabricación de acetaldehído [13]; biodiesel [14]; ácido monocloroacético [15]; epiclorohidrina [16]; deshidratación del propanol [17]; estireno [18] y ácido acrílico [19].

En el presente trabajo se lleva a cabo la simulación del proceso de producción de cumeno a partir de la alquilación del benceno con propileno utilizando el simulador Che$\mathrm{mCAD}^{\circledR}$ versión 5.2.0 [20], con el objetivo de conocer la composición másica de cada una de las corrientes involucradas en el proceso productivo, los principales parámetros de diseño de los equipos utilizados y el caudal requerido de los diferentes servicios auxiliares consumidos (vapor de agua, agua de enfriamiento, etc.) También se efectúa un estudio de sensibilidad para determinar la influencia de la presión de operación del tanque flash sobre la cantidad de cumeno a obtener por la corriente del fondo de este equipo.

\section{Materiales y Métodos}

\section{Propiedades físicas del cumeno}

El cumeno [iso-propilbenceno; 2-fenilpropano; (1-metiletil)benceno] presenta las propiedades físico-químicas que se muestran en la Tabla 1 [21]:

Tabla 1: Propiedades físico-químicas más importantes del cumeno

\begin{tabular}{|l|c|}
\hline Propiedad & Valor \\
\hline Fórmula molecular & $\mathrm{C}_{9} \mathrm{H}_{12} \mathrm{C}_{6} \mathrm{H}_{5} \mathrm{CH}\left(\mathrm{CH}_{3}\right)_{2}$ \\
\hline Peso molecular & 120,191 \\
\hline Punto de fusión & $-96,02^{\circ} \mathrm{C}$ \\
\hline Punto de ebullición & $152,41^{\circ} \mathrm{C}$ \\
\hline Densidad a $20^{\circ} \mathrm{C}$ & $0,8618 \mathrm{~g} / \mathrm{cm}^{3}$ \\
\hline Volumen molar a $20^{\circ} \mathrm{C}$ & 139,5 \\
\hline Entalpía de vaporización & $37,53 \mathrm{~kJ} / \mathrm{mol}^{\circ}$ \\
\hline Entalpía de fusión & $7,32 \mathrm{~kJ} / \mathrm{mol}$ \\
\hline Solubilidad en agua $\left(15-80^{\circ} \mathrm{C}\right)$ & $56 \mathrm{~g} / \mathrm{m}^{3}$ \\
\hline
\end{tabular}

\section{Descripción del proceso de producción}

La Figura 1 muestra el diagrama de flujo del proceso de producción del cumeN ${ }^{\circ}$ El benceno es alimentado al proceso con un caudal másico de $10545,39 \mathrm{~kg} / \mathrm{h}$, y a $25^{\circ} \mathrm{C}$ de temperatura y $1,3 \mathrm{~atm}$ de presión. A continuación es enviado hacia un tanque de mezclado, en donde se mezcla con una corriente de reciclo $\left(39,6^{\circ} \mathrm{C}, 1,28 \mathrm{~atm}\right)$ proveniente del tope de la primera columna de destilación (columna de benceno), la cual contiene fundamentalmente benceno, con trazas de cumeno, propileno y propaN $\mathrm{N}^{\circ} \mathrm{El}$ benceno reciclado incrementa la relación benceno/propileno en el reactor y consecuentemente minimiza la producción de di-isopropilbenceno (DIPB). A continuación, a la mezcla obtenida a la salida del tanque de mezclado se le incrementa la presión hasta alcanzar las $31 \mathrm{~atm}$, y es mezclada con una corriente de propileno $(6749,08 \mathrm{~kg} / \mathrm{h})$, la cual se encuentra a $27^{\circ} \mathrm{C}$ y $31 \mathrm{~atm}$, y contiene trazas de propano $(5,0 \% \mathrm{~m} / \mathrm{m})$. En este proceso se emplea una relación másica benceno/propileno de 2:1. La mezcla resultante obtenida es precalentada en un intercambiador de tubo y coraza, hasta alcanzar una temperatura de $232^{\circ}$ $\mathrm{C}$, para luego ser enviada hacia un calentador quemador, en donde se le incrementa la temperatura hasta alcanzar 
$\operatorname{los} 350^{\circ} \mathrm{C}$. La mezcla gaseosa calentada es enviada luego hacia el reactor, del tipo tubo y coraza y contiene ácido fosfórico como catalizador ácido soportado en diatomita. En este equipo ocurren las reacciones exotérmicas de transformación de los reactantes en productos, requiriéndose el uso de un agente de transferencia de calor (Dowtherm) para mantener la temperatura de reacción en el intervalo deseado $\left(350^{\circ} \mathrm{C}\right)$. Durante la reacción se obtiene una conversión del propileno del 99\% y una selectividad molar de cumeno/DIPB de 31:1. A la mezcla gaseosa reaccionante proveniente del reactor se le reduce la presión hasta los 2,2 atm, y seguidamente es enfriada en un intercambiador de tubo y coraza hasta los $65^{\circ} \mathrm{C}$, obteniéndose una mezcla bifásica vapor/líquido a la salida. El enfriador utiliza agua de enfriamiento como agente de intercambio de calor. Esta mezcla bifásica es enviada posteriormente hacia un tanque flash, donde se separan los gases de la porción líquida. Los gases separados en este recipiente, ricos en propileno y propano, son venteados a la atmósfera, aunque se recomienda utilizarlos como combustible para incrementar el valor añadido de los subproductos que se obtienen en el proceso de producción. La corriente líquida es enviada hacia la primera columna de destilación (columna de benceno), donde el benceno y otros componentes ligeros tales como el propileno y el propano remanentes son separados del cumeno y el DIPB. La corriente del tope $\left(101,7^{\circ} \mathrm{C}\right)$, rica en benceno es enfriada hasta $40^{\circ} \mathrm{C}$ por medio de un intercambiador de calor de tubo y coraza, y recirculada al proceso, mientras que la corriente del fondo $\left(188,3^{\circ} \mathrm{C}\right)$ es enviada hacia la segunda columna de destilación (columna de cumeno), en la cual se lleva a cabo la purificación del cumeno, esto es, su separación del DIPB. En esta columna se obtiene cumeno por el tope $\left(177,6^{\circ} \mathrm{C}\right)$ con una pureza del $99,9 \%$, mientas que el DIPB es obtenido por el fondo $\left(283,7^{\circ} \mathrm{C}\right)$ y puede ser comercializado como combustible. Debido a que las temperaturas de ebullición del propileno, benceno, cumeno y DIPB varían considerablemente entre sí, las separaciones de los componentes en las columnas de destilación se llevan a cabo fácil y eficientemente.

\section{Reacciones que ocurren en el reactor}

La Tabla 2 muestra las reacciones que ocurren en el reactor, asi como también su cinética de reacción [8], las cuales tienen lugar sobre un lecho fijo de gránulos de catalizador a una temperatura de $350^{\circ} \mathrm{C}$ y presiones que oscilan entre $25-32$ atm en fase de vapor.

Tabla 2: Cinética de reacción en el reactor

\begin{tabular}{|l|c|c|}
\hline Reacción & $\begin{array}{c}\text { Expresión de la } \\
\text { velocidad de } \\
\text { reacción }\end{array}$ & $\begin{array}{c}\text { Constantes de velocidad de } \\
\text { reacción }\end{array}$ \\
\hline$C_{3} H_{6}+C_{6} H_{6} \stackrel{k 1}{\longrightarrow} C_{9} H_{12}$ & $r_{\text {cumeno }}=k_{1} \cdot C_{B} \cdot C_{P}$ & $k_{1}=3,5 \cdot 10^{4} \exp \left(\frac{-24,9}{R T}\right)$ \\
\hline$C_{3} H_{6}+C_{9} H_{12} \stackrel{k 1}{\longrightarrow} C_{12} H_{18}$ & $r_{D I P B}=k_{2} \cdot C_{B} \cdot C_{C}$ & $k_{1}=2,9 \cdot 10^{6} \exp \left(\frac{-35,08}{R T}\right)$ \\
\hline
\end{tabular}

Donde la E está en $\mathrm{kcal} / \mathrm{mol}$; la concentración en $\mathrm{mol} / \mathrm{L}$, temperatura en Kelvin y la velocidad de reacción (r) en $\mathrm{mol} /$ (g cat.s)

Según se puede observar en la Tabla 2, la energía de activación de la reacción del cumeno (ecuación 1) es menor que la de formación del DIPB (ecuación 2). Por tanto, a menor temperatura del reactor mayor será la selectividad de la reacción del cumeno sobre la reacción del DIPB. Pero la menor temperatura de reacción traerá consigo una menor conversión por parte de la reacción. Por tanto deberá existir un compromiso entre la conversión por pase y la selectividad, por lo que se selecciona una temperatura de reacción de $350^{\circ} \mathrm{C}[22]$.

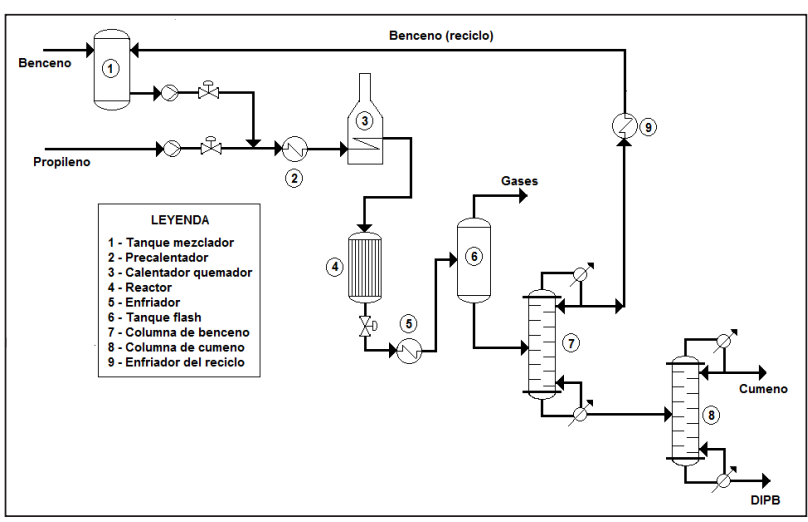

Figura 1: Diagrama de flujo del proceso de producción de cumeno a partir de la alquilación del benceno con propileN

\section{Propiedades el catalizador}

Según se describió con anterioridad, el catalizador empleado será ácido fosfórico soportado sobre diatomita, el cual presenta las siguientes propiedades [22] (Tabla 3):

Tabla 3: Propiedades del catalizador empleado

\begin{tabular}{|c|c|}
\hline Propiedad & Valor \\
\hline Diámetro de partícula $\left(\mathrm{d}_{\mathrm{p}}\right)$ & $3 \mathrm{~mm}$ \\
\hline Densidad de partícula $\left(\rho_{\text {cat }}\right)$ & $1600 \mathrm{~kg} / \mathrm{m}^{3}$ \\
\hline Fracción hueca $(\varepsilon)$ & 0,50 \\
\hline
\end{tabular}

\section{Características del simulador ChemCAD ${ }^{\circledR}$}

El simulador ChemCAD ${ }^{\circledR}$ es una herramienta poderosa y efectiva que presenta una interfaz gráfica de fácil manejo, mediante la cual se puede llevar a cabo la simulación de diferentes procesos e industrias tales como la farmacéutica, petroquímica, producción de compuestos químicos y biocombustibles, procesamiento de gases, entre otras. Posee una base datos de más de 2000 compuestos químicos entre sólidos, líquidos, gases y electrolitos, así como también varios modelos termodinámicos, tipos de equipamiento y unidades de ingeniería, lo cual permite la simulación tanto de sistemas en estado estacionario como dinámico. Puede ser empleada por los ingenieros para diseñar, controlar y optimizar nuevos procesos y/o plantas químicas, realizar 
estudios de sensibilidad, ejecutar balances de masa y energía, efectuar cálculos económicos, asi como también llevar a cabo estudios de escalado, con el objetivo de resolver problemas usualmente encontrados en las industrias, para así incrementar su productividad y el rendimiento.

\section{Modelo termodinámico empleado}

De acuerdo con los límites de temperaturas y presiones establecidas por el proceso, así como también debido a las propiedades físico-químicas de las compuestos químicos manejados, se escoge un modelo termodinámico del tipo Peng-Soave-Redlich-Kwong (PSRK) con asociación en fase de vapor, el cual se encuentra contenido en la base de datos de modelos termodinámicos del simulador Chem$\mathrm{CAD}^{\circledR}$. Vale destacar que el propio simulador ChemCAD ${ }^{\circledR}$ propuso el empleo del modelo termodinámico PSRK considerando las características del proceso productivo, mediante el uso de la opción "K-Value Wizard".

\section{Estudio de sensibilidad}

Se efectuó un estudio de sensibilidad para determinar la influencia de la presión de operación del tanque flash sobre la cantidad a obtener de cumeno por la corriente del fondo de este equipo, haciendo uso de la opción "Sensitivity Study" contenida en el simulador ChemCAD ${ }^{\circledR}$. Para ello se varió la conversión fraccional entre 1 - 10 atm, manteniendo constante la temperatura de operación de este equipo en $65^{\circ} \mathrm{C}$.

\section{Resultados y Discusión}

Diagrama de flujo del proceso de producción simulado en el ChemCAD ${ }^{\circledR}$

La Figura 2 muestra el diagrama de flujo obtenido una vez simulado el proceso productivo en el simulador ChemCAD ${ }^{\circledR}$.

\section{Resultados de las corrientes de proceso}

La Tabla 4 muestra los caudales másicos de cada una de las corrientes de entrada, intermedias y finales involucradas en el proceso, así como también sus temperaturas, presiones y fracción de vapor.

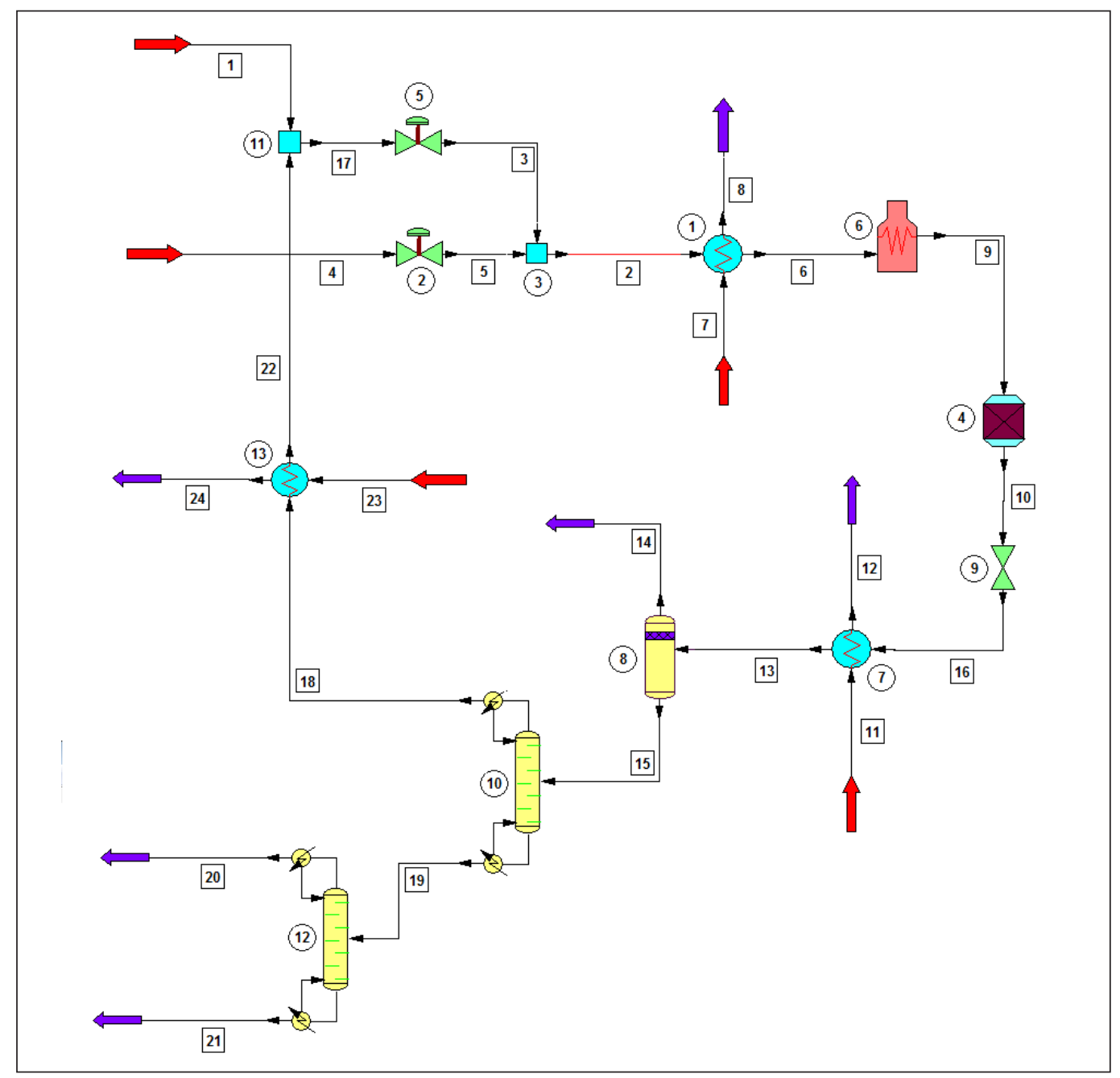

Figura 2: Diagrama de flujo del proceso de producción de cumeno una vez simulado en el simulador ChemCAD ${ }^{\circ}$ 
Tabla 4: Resultados obtenidos para las principales corrientes de proceso

\begin{tabular}{|c|c|c|c|c|c|}
\hline \multirow{2}{*}{$\begin{array}{c}\text { Variable } \\
1\end{array}$} & \multicolumn{5}{|c|}{ Número de la corriente (referirse a la Fig. 2) } \\
\hline & 17 & 22 & 4 & 3 & \\
\hline Temperatura $\left({ }^{\circ} \mathrm{C}\right)$ & 25,00 & 35,11 & 40,00 & 25,00 & 33,55 \\
\hline Presión (atm) & 1.3 & 1,28 & 1.9 & 1.3 & 31 \\
\hline Fracción de vapor & 0 & 0 & 0 & 0 & 0 \\
\hline Compuesto & \multicolumn{5}{|c|}{ Caudal [kg/h] } \\
\hline Propileno & - & 412,38 & 412,38 & 6411,63 & 412,38 \\
\hline Benceno & 10545,39 & 30296,60 & 19751,21 & - & 30296,60 \\
\hline Cumeno & - & 135,66 & 135,66 & - & 135,66 \\
\hline Propano & - & 434,39 & 434,39 & 337,45 & 434,39 \\
\hline DIPB & - & $8,10 \times 10^{-7}$ & $8,10 \times 10^{-7}$ & - & - \\
\hline Total & 10545,39 & 31279,03 & 20733,64 & 6749,08 & 31279,03 \\
\hline \multirow{2}{*}{ Variable } & \multicolumn{5}{|c|}{ Número de la corriente (referirse a la Fig. 2) } \\
\hline & 5 & 2 & 6 & 9 & 10 \\
\hline Temperatura $\left({ }^{\circ} \mathrm{C}\right)$ & 74,25 & 67,65 & 232 & 350 & 350 \\
\hline Presión (atm) & 31 & 31 & 30 & 29 & 28 \\
\hline Fracción de vapor & 1 & 0 & 1 & 1 & 1 \\
\hline \multicolumn{6}{|l|}{ Compuesto } \\
\hline Propileno & 6411,63 & 6824,00 & 6824,00 & 6824,00 & 750,63 \\
\hline Benceno & - & 30296,6 & 30296,6 & 30296,6 & 20274,19 \\
\hline Cumeno & - & 135,66 & 135,66 & 135,66 & 13584,27 \\
\hline Propano & 337,45 & 771,85 & 771,85 & 771,85 & 771,58 \\
\hline DIPB & - & $8,10 \times 10^{-7}$ & $8,10 \times 10^{-7}$ & $8,10 \times 10^{-7}$ & 2631,40 \\
\hline Total & 6749,08 & 38028,11 & 38028,11 & 38028,11 & 38012,07 \\
\hline \multirow{2}{*}{ Variable } & \multicolumn{5}{|c|}{ Número de la corriente (referirse a la Fig. 2) } \\
\hline & 16 & 13 & 14 & 15 & 18 \\
\hline Temperatura $\left({ }^{\circ} \mathrm{C}\right)$ & 331,37 & 65 & 65 & 65 & 102,7 \\
\hline Presión (atm) & 2,2 & 2 & 1,97 & 1,97 & 2 \\
\hline Fracción de vapor & 1 & 0,046 & 1 & 0 & 1 \\
\hline \multicolumn{6}{|l|}{ Compuesto } \\
\hline Propileno & 750,63 & 750,63 & 338,25 & 412,38 & 412,38 \\
\hline Benceno & 20274,19 & 20274,19 & 323,46 & 19950,72 & 19751,21 \\
\hline Cumeno & 13584,27 & 13584,27 & 18,43 & 13565,83 & 135,66 \\
\hline Propano & 771,58 & 771,58 & 337,19 & 434,39 & 434,39 \\
\hline DIPB & 2631,40 & 2631,40 & 0,0267 & 2631,37 & $8,10 \times 10^{-7}$ \\
\hline Total & 38012.07 & 38012.07 & 1017,36 & 36994,69 & 20733,64 \\
\hline \multirow{2}{*}{ Variable } & \multicolumn{5}{|c|}{ Número de la corriente (referirse a la Fig. 2) } \\
\hline & 19 & 20 & 21 & & \\
\hline Temperatura $\left({ }^{\circ} \mathrm{C}\right)$ & 188,70 & 177,63 & 283,76 & & \\
\hline Presión (atm) & 2,2 & 1,97 & 2,17 & & \\
\hline Fracción de vapor & 0 & 0 & $1,53 \times 10^{-6}$ & & \\
\hline \multicolumn{6}{|l|}{ Compuesto } \\
\hline Propileno & - & - & - & & \\
\hline Benceno & 199,51 & 165,17 & 34,34 & & \\
\hline Cumeno & 13430,18 & 13403,54 & 26,64 & & \\
\hline Propano & - & - & - & & \\
\hline DIPB & 2631,37 & 26,53 & 2604,84 & & \\
\hline Total & 16261,06 & 13595,24 & 2665,82 & & \\
\hline
\end{tabular}


Como bien se puede percibir en la Tabla 4, la corriente de salida del reactor (equipo $\mathrm{N}^{\circ} 10$ en la Fig. 2) contiene mayormente benceno $(53,3 \%)$ y cumeno $(35,7 \%)$. La relación cumeno/DIPB en esta corriente es de 5:1 aproximadamente, mientras que el $89,0 \%$ del propileno que entró al reactor se convirtió en cume $\mathrm{N}^{\circ} \mathrm{A}$ continuación, en el tanque flash $\left(\mathrm{N}^{\circ} 8\right)$, la corriente del tope de este equipo contiene fundamentalmente propileno $(33,2 \%)$, benceno $(31,7 \%)$ y propano $(33,1 \%)$; mientras que la corriente del fondo trae consigo fundamentalmente benceno $(53,9 \%)$ y cumeno $(36,7 \%)$, con pequeñas cantidades de DIPB $(7,1 \%)$, propano $(1,2 \%)$ y propileno $(1,1 \%)$. En la columna de benceno $\left(\mathrm{N}^{\circ} 10\right)$ se obtiene por el tope una corriente compuesta mayormente por benceno $(95,3 \%)$, la cual es recirculada de vuelta al proceso. La corriente del fondo de este equipo, por su parte, contiene esencialmente cumeno $(82,6 \%)$ y DIPB (16,2\%). Por último, la corriente del tope de la columna de cumeno $\left(\mathrm{N}^{\circ} 12\right)$ incluye mayormente cumeno $(13403,54 \mathrm{~kg} / \mathrm{h})$ con una pureza del $98,6 \%$, con trazas de benceno $(1,2 \%)$ y DIPB $(0,2 \%)$, la cual puede considerarse de aceptable considerando que este es el producto final deseado por el proceso, mientras que la corriente del fondo de esta columna contiene principalmente DIPB con un $99,7 \%$ de pureza. De forma general, la cantidad total de cumeno que se pierde por las corrientes del tope del tanque flash y la del fondo de la columna de cumeno (sin incluir el reciclo) es baja, alcanzando un valor de $45,07 \mathrm{~kg} / \mathrm{h}$, incrementando de esta manera el rendimiento global del proceso.

\section{Equipamiento}

\section{Intercambiadores de calor}

La Figura 3 muestra el calor intercambiado (Q), la Media Logarítmica de la Diferencia de Temperatura (MLDT) calculada, el área total de transferencia de calor (A) y el caudal másico de servicio auxiliar demandado para cada uno de los intercambiadores de calor utilizados en el proceso.

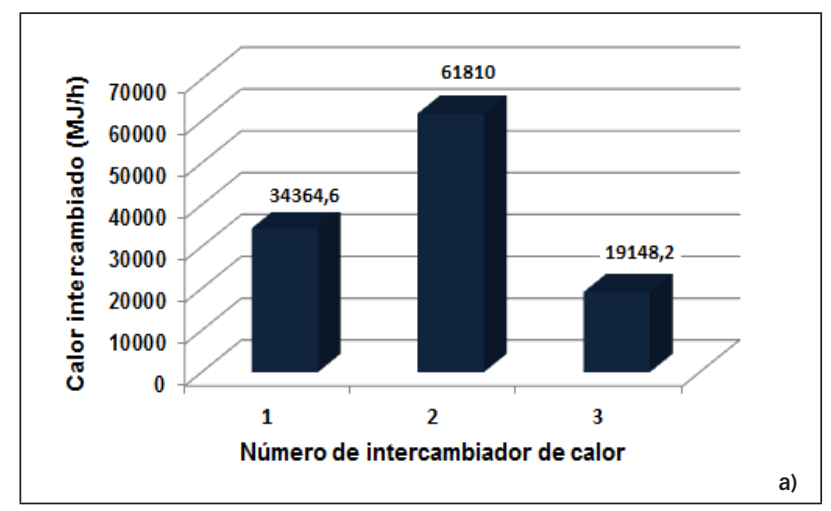

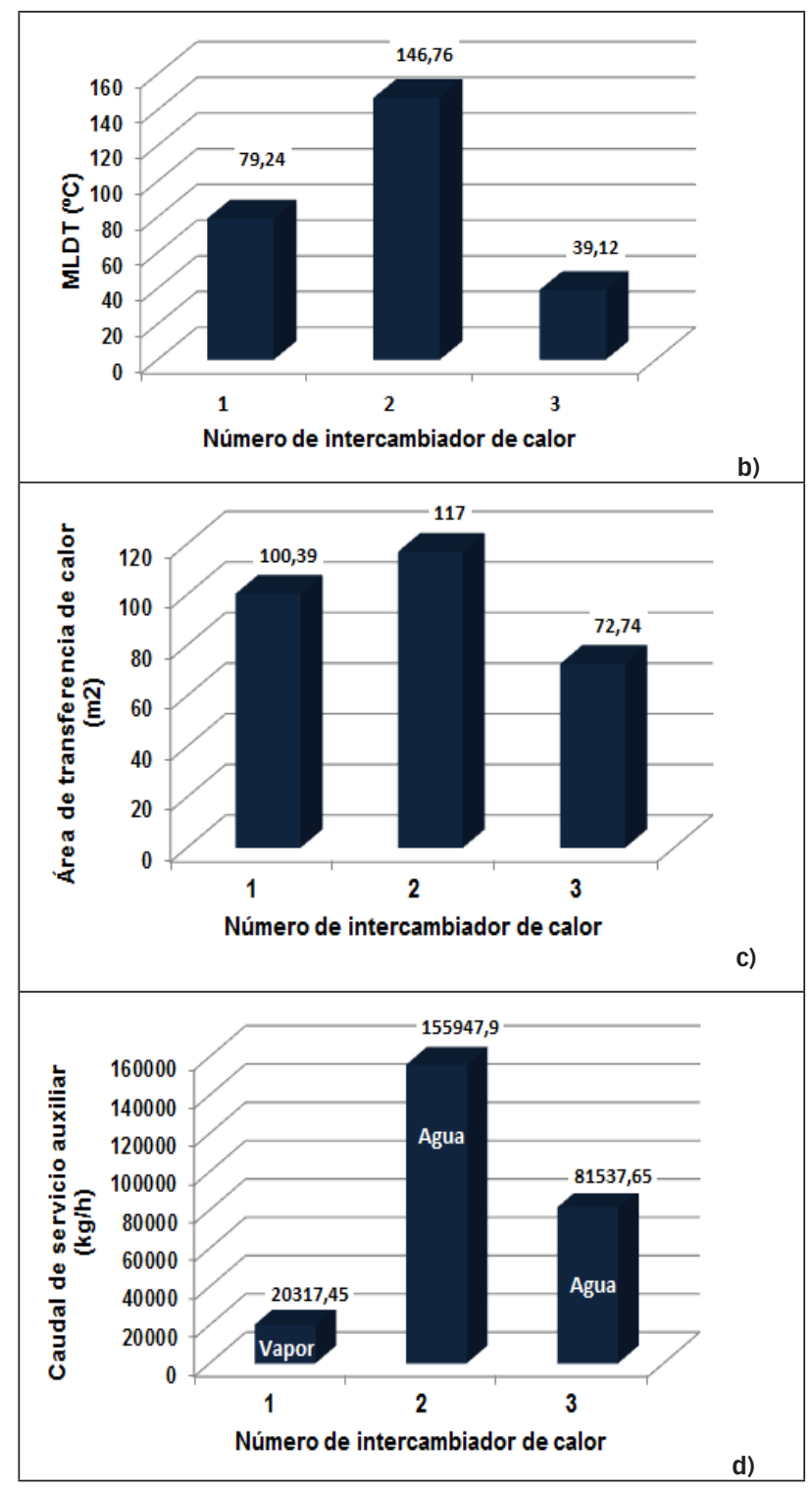

Figura 3: Resultados obtenidos para los intercambiadores de calor. a) Calor intercambiado. b) MLDT. c) Área de transferencia de calor requerida. d) Caudal de servicio auxiliar.

De acuerdo con los resultados mostrados en la Figura 3, el segundo intercambiador de calor (equipo $\mathrm{N}^{\circ} 7$ en la Fig. 2) es el que más calor intercambia $(61810 \mathrm{MJ} / \mathrm{h})$, lo cual se debe fundamentalmente a que en este equipo es donde se le reduce la temperatura de la mezcla gaseosa reaccionante de salida del reactor desde $331^{\circ} \mathrm{C}$ hasta $65^{\circ} \mathrm{C}$, es decir, presenta un $\Delta \mathrm{T}$ elevado $\left(266^{\circ} \mathrm{C}\right)$, lo cual influye además en que sea el equipo que mayor MLDT posee $\left(146,76^{\circ} \mathrm{C}\right)$, el de mayor área de transferencia de calor requerida $\left(117 \mathrm{~m}^{2}\right)$, y el que mayor caudal de servicio auxiliar necesita, con $155947,9 \mathrm{~kg} / \mathrm{h}$ de agua de enfriamiento.

\section{Curvas de calor}

La Figura 4 muestra las curvas de calor obtenidas para cada uno de los intercambiadores de calor de tubo y coraza empleados en el proceso. 


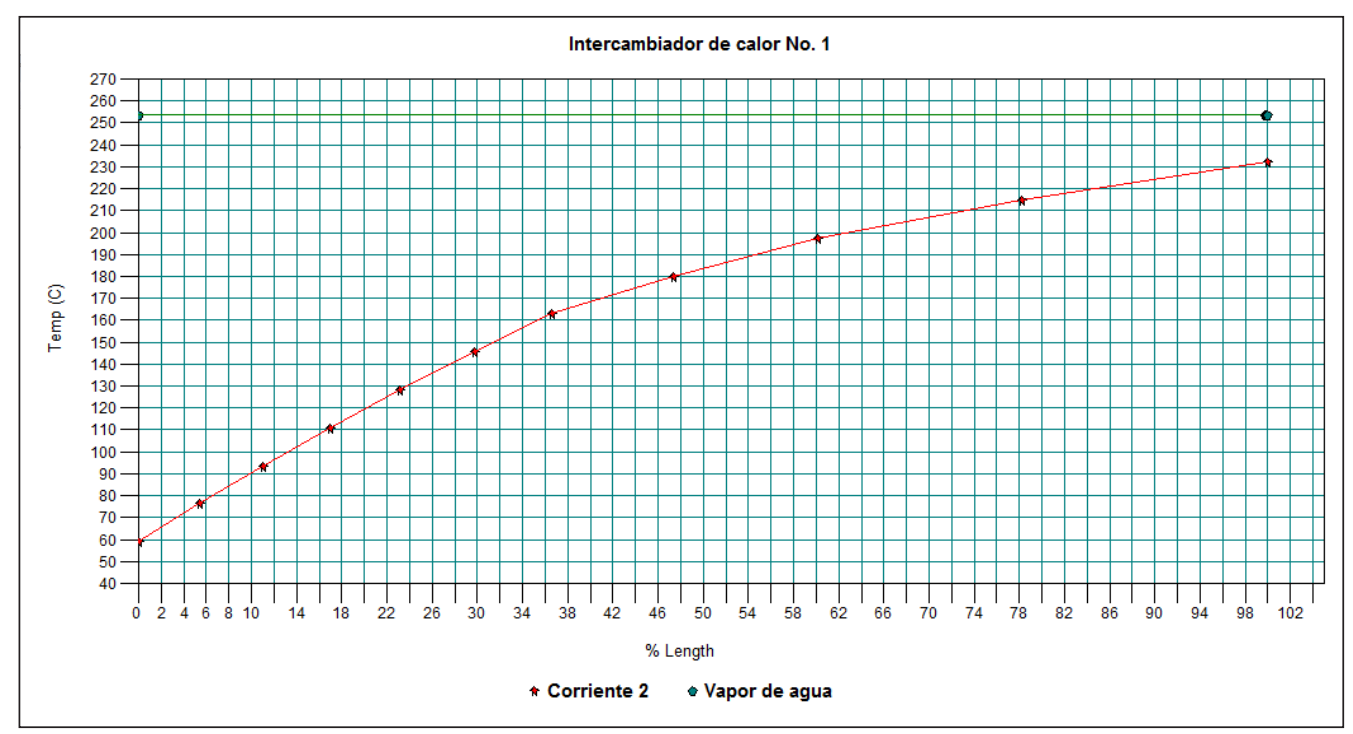

a)

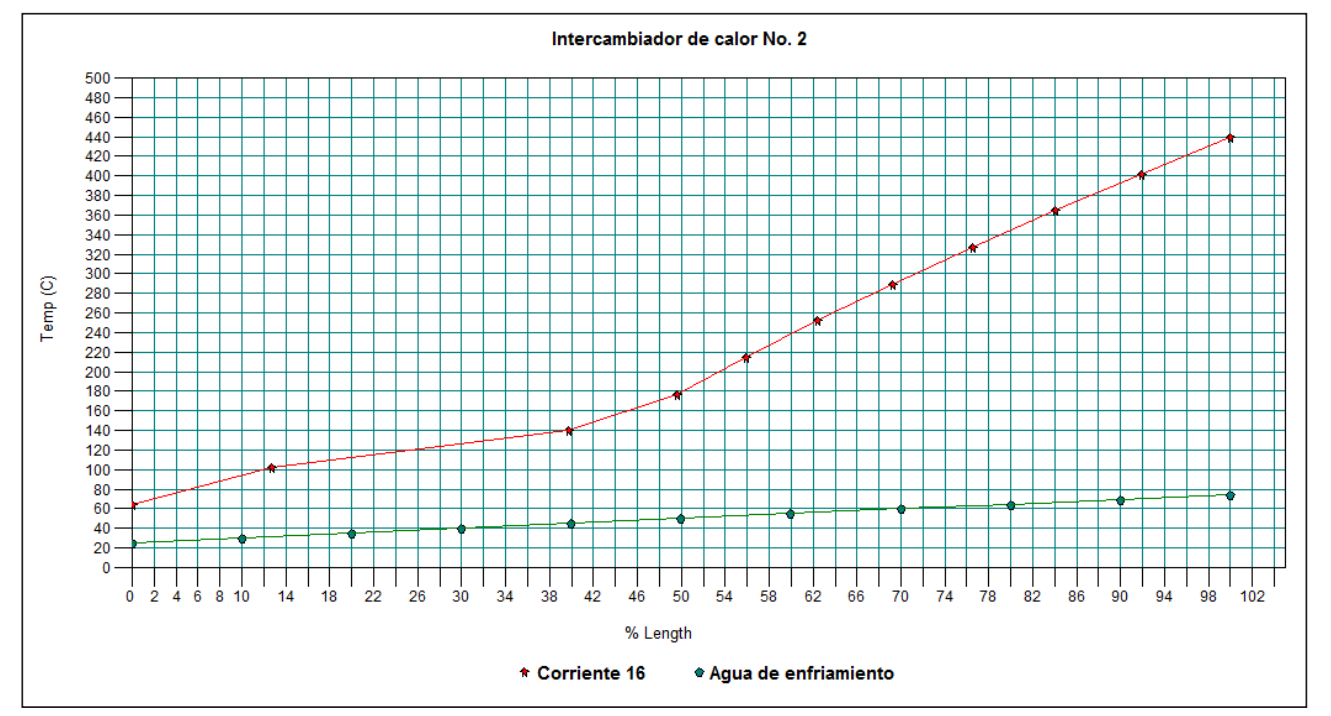

b)

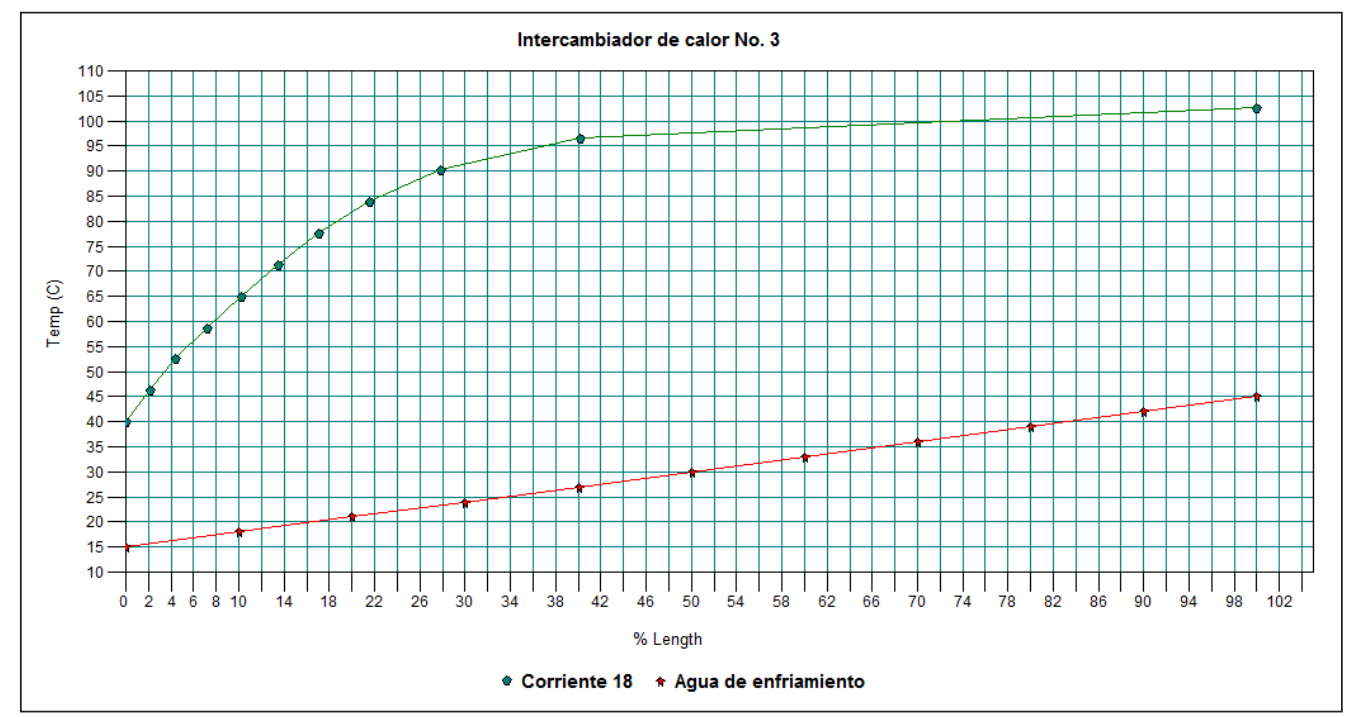

c)

Figura 4: Curvas de calor de cada intercambiador de calor de tubo y coraza utilizado en el proceso 
Según los resultados descritos en la Figura 4, la curva de calor obtenida para el intercambiador de calor $\mathrm{N}^{\circ} 1$ (equipo número 1 en la Figura 2) presenta un comportamiento lineal creciente hasta un $36 \%$ aproximadamente de la posición del intercambiador de calor, en donde tiene lugar un punto de inflexión a una temperatura de $162^{\circ} \mathrm{C}$, lo cual representa la ocurrencia de un cambio de fase (vaporización) de la mezcla líquida. A partir de ese punto, la curva de calor muestra un comportamiento lineal creciente también hasta alcanzar la temperatura final deseada $\left(232^{\circ} \mathrm{C}\right)$.

Por su parte, la curva de calor obtenida para el intercambiador de calor $\mathrm{N}^{\circ} 2$ (equipo número 7) presenta una tendencia lineal decreciente hasta alcanzar el $40 \%$ de la longitud del intercambiador, en cuya posición manifiesta un punto de inflexión que significa que existe un cambio de fase (condensación) de la mezcla gaseosa de salida del reactor, a una temperatura de $140^{\circ} \mathrm{C}$ aproximadamente.

Por último, la curva de calor obtenida para el tercer intercambiador de calor (equipo $\mathrm{N}^{\circ} 13$ ) muestra una tendencia lineal decreciente hasta llegar al $40 \%$ de la longitud del intercambiador (temperatura de $96^{\circ} \mathrm{C}$ ), en donde acontece un punto de inflexión que significa la ocurrencia de un cambio de fase (condensación) de la mezcla gaseosa de salida del tope de la columna de bence ${ }^{\circ}$ A partir de este punto la curva muestra una tendencia curvada decreciente hasta llegar a la temperatura de enfriamiento deseada $\left(40^{\circ} \mathrm{C}\right)$.

\section{Columnas de destilación}

La Tabla 5 expone los parámetros de diseño de ambas columnas de destilación calculados por el simulador ChemCAD $^{\circledR}$.
Tabla 5: Parámetros de diseño calculados por el simulador ChemCAD para ambas columnas de destilación.

\begin{tabular}{|l|c|}
\hline \multicolumn{1}{|c|}{ Parámetro } & Valor \\
\hline \multicolumn{2}{|c|}{ Columna de benceno } \\
\hline Carga de calor condensador (MJ/h) & $-10678,8$ \\
\hline Carga de calor rehervidor (MJ/h) & 24330,6 \\
\hline Etapas mínimas & 17 \\
\hline Etapa de alimentación & 6 \\
\hline Relación de reflujo, mínima & 0,254 \\
\hline Relación de reflujo & 1,25 \\
\hline & \\
\hline Carga de calor condensador (MJ/h) & $-9498,61$ \\
\hline Carga de calor rehervidor (MJ/h) & 9938,67 \\
\hline Etapas mínimas & 15 \\
\hline Etapa de alimentación & 5 \\
\hline Relación de reflujo, mínima & 0,139 \\
\hline Relación de reflujo & 1,30 \\
\hline
\end{tabular}

Tomando en cuenta los resultados mostrados en la Tabla 5, la columna de benceno deberá poseer un número total de platos o etapas mínimas de 17, siendo la etapa de alimentación la número seis. Por su parte, la columna de cumeno deberá poseer 15 etapas como mínimo, siendo la cinco la etapa o plato de alimentación.

\section{Resultados del estudio de sensibilidad}

La Figura 5 describe los resultados del estudio de sensibilidad efectuado.

Según se puede observar en la Figura 5, la cantidad de cumeno a obtener por la corriente de fondo del tanque flash se incrementa de forma constante hasta los 6,4 atm aproximadamente, para luego presentar un comportamiento estacionario a partir de este valor de presión. Debido a esto se aconseja operar el tanque flash a una presión de 7 atm para obtener el rendimiento máximo posible de cumeN ${ }^{\circ}$

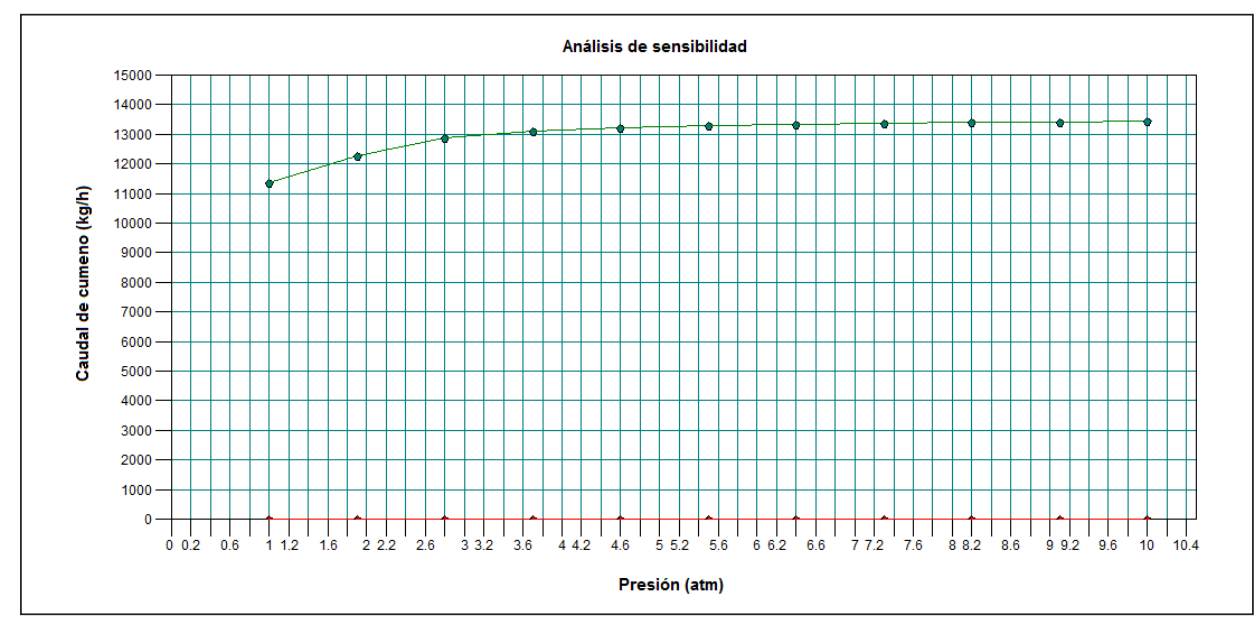

Figura 5: Resultados del estudio de sensibilidad efectuado 


\section{Conclusiones}

El empleo del simulador ChemCAD ${ }^{\circledR}$ permitió llevar a cabo la simulación exitosa del proceso de producción de cumeno a partir de la alquilación del benceno con propileN ${ }^{\circ}$

Se obtiene cumeno por la corriente del tope de la columna de destilación $\mathrm{N}^{\circ} 2$ (columna de cumeno) con un caudal de $13403,54 \mathrm{~kg} / \mathrm{h}$ y una pureza del 98,6\%, lo cual puede considerarse de aceptable.

Las curvas de calor obtenidas de cada intercambiador de calor permiten conocer y analizar el perfil de temperatura seguido por cada corriente en dependencia de la longitud del equipo.

El intercambiador de calor $\mathrm{N}^{\circ} 2$ presenta la mayor carga calórica (61 $810 \mathrm{MJ} / \mathrm{h}$ ), el mayor valor de MLDT $\left(146,76^{\circ} \mathrm{C}\right)$, la mayor área de transferencia de calor requerida $\left(117 \mathrm{~m}^{2}\right)$ y el mayor caudal de servicio auxiliar necesario (155 947,9 kg/h de agua de enfriamiento), lo cual se debe a que es el intercambiador que mayor $\Delta \mathrm{T}$ presenta $\left(266^{\circ} \mathrm{C}\right)$.

Se recomienda operar el tanque flash a una presión de 7 atm para maximizar la cantidad de cumeno a obtener por la corriente del fondo de este equipo.

\section{Referencias}

1. Speight, J. Chemical and process design handbook. McGraw-Hill, New York, p. 2.183 - 2.184. 2002.

2. Mahapatra, N. Design and simulation of cumene plant using Aspen Plus. (Bachelor of Technology in Chemical Engineering), National Institute of Technology Rourkela, India. 2010.

3. Panming, J.; Qiuying, w.; Chao, z., y Yanhe, x. Alkylation of benzene with propene to produce cumene over a nickel/yalumina catalyst. Applied Catalysis A: General, 91: p. 125-129. 1992.

4. Geatti, A.; Lenarda, M.; Storaro, L.; Ganzerla, R. y Perissinotto, M. Solid acid catalysts from clays: Cumene synthesis by benzene alkylation with propene catalyzed by cation exchanged aluminum pillared clays. Journal of Molecular Catalysis A: Chemical, 121: p. 111-118. 1997.

5. Shutkina, 0. v.; Ponomareva, O. A. y Ivanova, I. I. Catalytic Synthesis of Cumene from Benzene and Acetone. Petroleum Chemistry, 53(1): p. 20-26. 2013.

6. Jin, F.; Fan, M.-h.; Jia, Q.-f. y Li, Q.-x. Synthesis of Cumene from Lignin by Catalytic Transformation. Chinese Journal of Chemical Physics, 30(3): p. 348-356. 2017.

7. Luyben, w. L. Design and Control of the Cumene Process. Ind. Eng. Chem. Res., 49: p. 719-734. 2010.

8. Norouzi, H. R., y Fatemi, s. Economic Optimization of the Cumene Production Process Using Response Surface Methodology. Chem. Eng. Comm., 199: p. 1375-1393. 2012.

9. Gera, V.; Kaistha, N.; Panahi, M. y Skogestad, s. Plantwide Control of a Cumene Manufacture Process. Paper presented at the 21st European Symposium on Computer
Aided Process Engineering - ESCAPE 21. 2011.

10. Skogestad, s. Control structure design for complete chemical plants. Comp. Chem. Engg., 28: p. 219-234. 2004.

11. Ernst, S.; Garro, O. A.; Winkler, S.; Venkataraman, G.; Langer, R.; Cooney, C. L. y Sasisekharan, R. Process Simulation for Recombinant Protein Production: Cost Estimation and Sensitivity Analysis for Heparinase I Expressed in Escherichia coli. Biotechnology and Bioengineering, 53(6): p. 575-582. 1997.

12. Auli, N. A.; Sakinah, M.; Bakri, A. M. M. A.; Kamarudin, H. y Norazian, M. N. Simulation Of Xylitol Production: A Review. Australian Journal of Basic and Applied Sciences, 7(5): p. 366-372. 2013.

13. Eliasson, J. Design of an Plant for Manufacturing of Acetaldehyde. Lund, Sweden: Department of Chemical Engineering, Lund University. P. 12-37. 2010.

14. Chilev, C. y Simeonov, E. Simulation of Biodiesel Production by Transesterification of Vegetable Oils. Journal of Chemical Technology and Metallurgy, 49(5): p. 479486. 2014.

15. Greeshma, N.; Shah, B. H. y Patel, N. M. Simulation Study of Reactive Distillation for Monochloroacetic Acid using CHEMCAD. International Journal of Futuristic Trends in Engineering and Technology, 1(2): p. 1-11. 2014.

16. Almena, A. y Martín, M. Technoeconomic Analysis of the Production of Epichlorohydrin from Glycerol. Industrial \& Engineering Chemistry Research, 55: p. 3226-3238. 2016.

17. Wyczesany, A. Simulation of N-Propanol Dehydration Process via Heterogeneous Azeotropic Distillation Using the NRTL Equation. Chemical and Process Engineering, 38(1): p. 163-175. 2017.

18. Pérez, A.; Pérez, E. J. y Segura, R. M. Simulation of the styrene production process via catalytic dehydrogenation of ethylbenzene using CHEMCAD ${ }^{\circledR}$ process simulator. Tecnura, 21(53): 15-31. 2017.

19. Pérez, A.; Pérez, E. J. y Segura, R. M. Simulation of the acrylic acid production process through catalytic oxidation of gaseous propylene using Chem $C A D \circledR$ simulator. Ingeniare. Revista chilena de ingeniería, 27(1): p. 142-150. 2019.

20. Chemstations. (2002). ChemCAD ${ }^{\circledR}$ (Version 5.2.0). Wesel, Germany.

21. Mackay, D.; Shiu, w. Y.; Ma, K.-C. y Lee, s. C. Handbook of physical-chemical properties and environmental fate for organic chemicals (2nd ed. Vol. I. Introduction and Hydrocarbons). CRC Press, Boca Raton. p 500-505. 2006.

22. wvu. Design of a New, 100,000 Metric Ton Per Year, Cumene Production Facility West Virginia University: West Virginia, USA. 2012. 